\title{
Cambios en la estructura cuticular Ornithodoros erraticus hembra durante el proceso de alimentación
}

\section{Changes in cuticular structure of female Ornithodoros erraticus during the feeding process}

\author{
Marina Patricia Arrieta ${ }^{1,2 *}$, Santiago Ferrándiz ${ }^{1}$, Emilio Rayón ${ }^{3}$ \& Juan López ${ }^{1}$ \\ 1Instituto de Tecnología de Materiales. Universitat Politècnica de Valencia, 03801 Alcoy-Alicante. Spain. \\ ${ }^{2}$ Facultad de Ciencias Agropecuarias. Universidad Católica de Córdoba Camino a Alta Gracia Km 71⁄2, 5017 Córdoba, Argentina \\ ${ }^{3}$ Instituto de Tecnología de Materiales. Universitat Politècnica de Valencia, Cami de Vera s/n. 46022. Valencia \\ *Corresponding author. Tel.: +34-966528433; fax: +34-966528478 \\ E-mail address: marrieta@itm.upv.es (M.P. Arrieta)
}

\begin{abstract}
RESUMEN
Los ácaros ectoparásitos son parásitos que aumentan su tamaño en varios órdenes de magnitud cuando se alimentan y alcanzan su estado adulto. Esclarecer los mecanismos por los cuales una cutícula rígida puede adaptarse a un aumento de volumen importante, cobra importancia para el diseño de fórmulas que bloquen dicho crecimiento y por lo tanto el desarrollo de estos parásitos, y como modelo para el desarrollo de nuevos biomateriales. En este trabajo se realizó una investigación morfológica y química de la cutícula de ácaros Ornithodoros erraticus hembra en estado adulto en ayunas y alimentadas. Mediante imágenes de microscopía electrónica de barrido se muestra que la morfología en forma de pliegues permite un estiramiento de la cutícula en todos los ejes del plano cuticular, lo que indica que la cutícula se adapta al crecimiento del parásito durante su alimentación. Por otro lado, los resultados de un análisis de la composición química mediante pirólisis acoplada a cromatografía de gases y con detección por espectrometría de masas muestran pocas diferencias entre el estado en ayunas y alimentado. La molécula de indol, principal producto de pirólisis del aminoácido triptófano, podría ser indicativo de la pérdida de rigidez debido a que ese encontró mayoritariamente en la especie en ayunas y en mucha menor proporción en la especie alimentada.
\end{abstract}

Palabras Clave: Ornithodoros erraticus, ácaro, cutícula.

\begin{abstract}
Ectoparasite acari increases their size several orders of magnitude during feeding. Find out the mechanism through which the young stiff cuticle increases its volume, could be useful to develop novel formulations that would serve to block the growth of these parasites and also as a model for the development of new smart materials. In this work, morphological and chemical studies of the cuticle extracted from female Ornithodoros erraticus were carried out under two states; as a young unfed state and adult fed. SEM Micrographs show that the folds morphology allows stretching the cuticle at all plane axes, revealing that the cuticle is adapted to the parasite's growth during its feeding. On the other hand, chemical composition analysis by pyrolysis coupled to gas chromatography and mass spectrometry showed few changes between acari into unfed state and fed state adult. Indol, the main pyrolysis product of the amino acid tryptophan, could be indicative of the loss of rigidity due it was found mainly in the fasted species than in the fed ones.
\end{abstract}

KeYwords: Ornithodoros erraticus, acari, cuticle.

\section{INTRODUCCIÓN}

Las garrapatas son ácaros ectoparásitos temporales obligados capaces de parasitar vertebrados (Encinas Grandes et al. 1999b, Parola \& Didier 2001). En Europa son consideradas como el grupo de artrópodos hematófagos de mayor importancia en la transmisión de agentes transmitidos por vectores, siendo superados a nivel mundial, únicamente por los mosquitos (Barandika Iza 2010). Aunque favorecidas por clima tropical, se encuentran extendidas por todo el planeta (Parola \& Didier 2001), siendo además muy problemáticas en zonas de inviernos fríos, como por ejemplo en Canadá. 
Representan uno de los grandes problemas de salud pública y veterinaria en el mundo debido al gran número de enfermedades que son capaces de transmitir (Alonso-Diaz et al. 2006) tanto a animales como al hombre (Muñoz \& Casanueva 2001). Las garrapatas tanto de uno como de otro sexo presentan tres etapas básicas de la vida, pasando en su ciclo biológico por las fases de larva, ninfa y adulta (Encinas Grandes et al. 1999c, Parola \& Didier 2001). Existen más de ochocientas especies divididas en dos grandes familias: Ixodidae o "garrapatas duras" y Argasidae o "garrapatas blandas" (Encinas Grandes et al. 1999b,Parola \& Didier 2001). Una tercera familia, la Nuttalliellidae se representa por una única especie Nutalliella namaqua limitada al sur de África (Hackman 1982,Encinas Grandes et al. 1999b,Parola \& Didier 2001). El género Ornithodoros de la familia Argasidae es el más numeroso y el más importante como parásito de muchas especies (Iannacone \& Ayala 2004). Las garrapatas Argasidae presentan cutícula flexible (Parola \& Didier 2001), incluso en su estado de ayunas (Kaufman et al. 2010), y principalmente se distinguen de las Ixodidae por carecer de escudo dorsal esclerotizado (Encinas Grandes et al. 1999c). Debido a su gran tamaño, al menos en su estado adulto, resultan visibles a simple vista (Encinas Grandes et al. 1999b). En este sentido, las hembras adultas una vez que copulan pueden alcanzar hasta 100 veces el peso de su cuerpo sin alimentar (Flynn \& Kaufman 2011). Presentan una característica particular, que las diferencia de otros artrópodos, el gran tamaño que adquieren durante el proceso de alimentación, debido a su capacidad de aumentar la extensibilidad de la cutícula (Dillinger \& Kesel 2002). La morfología y biología de las garrapatas resultan muy uniformes, siendo consideradas como organismos conservadores, sin presentar apenas desviaciones respecto al modelo que se estableció en las formas ancestrales de las que derivan (Encinas Grandes et al. 1999b). La cutícula está formada por la epicutícula (estrato superficial) y la procutícula (endocutícula y exocutícula). La epicutícula es la que contribuye en mayor medida a la impermeabilización de la cutícula. En la procutícula, compuesta principalmente por quitina y proteínas, los homopolímeros lineales de $\beta(1$ 4)N-acetilglucosamina (NAG) se unen entre sí formando microfibrillas y las proteínas formando complejos glucoprotéicos, según se muestra en la Figura 1. La rigidez de la cutícula se debe a la unión de las proteínas a la quitina en la exocutícula mediante un proceso de esclerotización donde la tirosina se transforma en N-acetildopamina, la cual actúa como molécula de unión entre aminoácidos de proteínas adyacentes (Encinas Grandes 1999a). Estos ácaros durante su desarrollo juvenil cambian varias veces de cutícula (ecdisis) sustituyéndola por una nueva más grande con la finalidad de posibilitar el desarrollo corporal. La rotura de uniones intermoleculares no covalentes, precede a la rápida expansión de las cutículas (Hackman $\&$ Goldberg 1987). Durante el momento de la ecdisis, la cutícula nueva se plastifica, es decir adquiere la capacidad de deformarse más fácilmente en respuesta a una fuerza aplicada. La plastificación, es una adaptación de la cutícula para reducir la energía metabólica necesaria para poder ampliarse (Kaufman et al. 2010). Durante esta etapa experimentan cambios bruscos de forma, metamorfosis, hacia la fase adulta (Encinas Grandes 1999a). Kaufman y col., (2010) estudiaron la plastificación de garrapatas, de las familias Argasidae (Ornithodoros moubata) y Ixodidae (Amblyomma hebraeum), sin alimentar y alimentadas. En el caso de las Ixodidae no se observó relación entre el proceso de alimentación y la plastificación cuticular, debido a que se deposita gran cantidad de endocutícula nueva durante el proceso de alimentación. Mientras que para la familia Argasidae, encontraron que el mecanismo de hidratación durante el proceso de alimentación produce plastificación cuticular debida al aumento de la acidez producida poco después del comienzo de la alimentación (Kaufman et al. 2010). De esta manera, la plastificación reversible de la cutícula durante la alimentación se puede controlar por la variación del pH en la cutícula (Lomakin et al. 2011).

Para estudiar el comportamiento de muestras biológicas a escala micrométrica se debe comenzar con la observación de la muestra mediante microscopía óptica. Una vez conocidas sus características generales, la técnica de microscopía electrónica de barrido nos permite obtener información detallada de una zona de la muestra a niveles microscópicos (Ojeda Sahagún 1997). Dado el alto vacío requerido en un microscopio de barrido electrónico se requiere del tratamiento por criodesecación del ejemplar biológico. Con este tratamiento se reduce al mínimo las deformaciones producidas por otras técnicas como la desecación, especialmente las debidas a la tensión superficial. De este modo, solidificando el líquido de la muestra por vacío se consigue eliminar los efectos de la tensión superficial al eliminar la interfase líquido-gas (Ojeda Sahagún 1997). Por otra parte, también resulta significativo estudiar los compuestos químicos que componen un material. Para ello la combinación de la técnica de pirólisis acoplada a cromatografía de gases y con detección por espectrometría de masas (Py-GC/MS) es una técnica analítica muy sensible que permite identificar los compuestos producidos durante la degradación térmica (Arrieta et al. 2012). En este sentido, la técnica de Py-GC/MS ha sido aplicada con éxito para el estudio de macromoléculas, incluyendo lignina, celulosa, proteínas y quitina, en diversos tejidos biológicos (Stankiewicz et al. 1998).

En el presente trabajo se ha realizado una caracterización morfológica y química de ácaros Ornithodoros erraticus hembra en estado de ayunas y en estado alimentado mediante microscopía óptica, microscopía de barrido electrónico. Se pretende aclarar qué le ocurre morfológicamente a la cutícula 
del ácaro cuando éste aumenta su tamaño al alimentarse. Además se pretende realizar un estudio químico mediante pirolisis Py-GC/MS con el fin de detectar algún posible cambio bioquímico entre ambos estados, como estudio preliminar para la generación de posibles bloqueantes de crecimiento y para entender el mecanismo de adaptación de la cutícula con el objeto de valorar posibles aplicaciones mediante biomímesis.
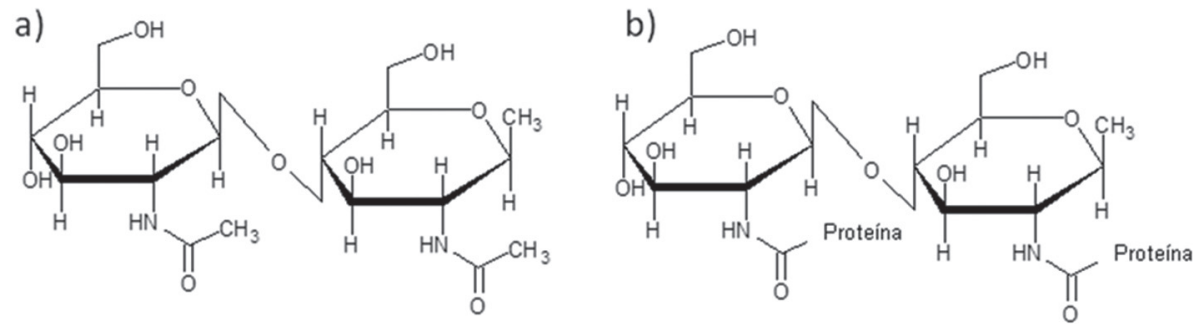

Figura 1. Representación gráfica de las moléculas de a) Quitina y b) complejo glucoprotéico.

Figure 1. Graphic representation of molecules of a) Chitin and b) glucoprotein complex.

\section{MATERIALES Y MÉTODOS}

Se estudiaron especímenes de Ornithodoros erraticus hembras adultas en estado de ayunas y alimentada. Los mismos fueron obtenidos de una población representativa de 25 individuos en su estado de ayunas en el área de Salamanca (España) en huecos de porqueras de cerdos. Para el estudio del estado alimentado, estos ejemplares fueron alimentados sobre conejos en laboratorio, con la finalidad de que todos los ejemplares tengan el mismo tipo y tiempo de alimentación ya que el hospedero puede jugar un rol en el ciclo reproductivo de los ácaros (Muñoz et al. 2003). Todos los ejemplares fueron recogidos y mantenidos en etanol al 97\% durante varios días antes de comenzar el estudio (Berrios 2002). Previo a cada análisis las muestras fueron secadas en aire a temperatura ambiente durante 24 horas. Se determinaron medidas de longitud (1), anchura (w) y altura (e) mediante el uso de un calibre. El peso de las muestras (m) se registró mediante el uso de una balanza electrónica (Mettler Toledo) con precisión de 0,1 mg. Se calculó el volumen del cuerpo según la Ecuación 1 (Flynn \& Kaufman 2011):

$$
V=\frac{4}{3} \times \pi \times\left(\frac{l}{2} \times \frac{w}{2} \times \frac{e}{2}\right) \quad \text { Ecuación } 1
$$

La caracterización visual se llevó a cabo mediante microscopía óptica y microscopía electrónica de barrido. Se utilizó una lupa binocular de Leica Microsystems modelo MZ APO. Las imágenes de microscopio óptico se obtuvieron con la técnica Extended Depth of Focus, EDF mediante un microscopio de luz reflejada Nikon LV-100 y el software NIS-Elements. La técnica EDF permite capturar imágenes en diferentes planos focales para posteriormente generar una única imagen enfocada o una superficie 3D.

Para la observación en el microscopio electrónico de barrido se utilizó un JEOL-JSM5410 preparado para la observación de muestras criofijadas (crio-SEM). En todos los casos las estructuras fueron fijadas mediante criofijación a $-180^{\circ} \mathrm{C}$ en nitrógeno líquido. Tanto la sublimación del hielo superficial como el recubrimiento de oro mediante la técnica de sputtering se realizaron en el interior del microscopio. Para la observación de secciones, se realizó un corte en dos etapas: (i) a temperatura ambiente mediante corte con bisturí se diseccionaron longitudinalmente. (ii) Una vez la muestra fue fijada a $-180^{\circ} \mathrm{C}$ y anterior al proceso de metalizado con oro, se realizó un segundo corte por fractura frágil perpendicular a la sección. De este modo se aseguró el preservar las estructuras internas de la cutícula.

Para la pirólisis de las muestras se utilizó un pirolizador (Py) "Pyroprobe 1000, CDS Analytical", (Oxford, Pensilvania) equipado con una interface (CDS 1500) con inyector manual. El pirolizador fue acoplado a un cromatógrafo de gases (GC) "6890N, Agilent Technologies", (España S.L., Madrid, España). Se empleó una columna cromatográfica HP-5 $\mathrm{ms}$ (30 m de longitud; $0,25 \mathrm{~mm}$ de espesor y con una fase estacionaria de $0,25 \mu \mathrm{m})$. El horno del GC fue programado a $40^{\circ} \mathrm{C}$ durante 2 minutos seguido de una rampa a $5^{\circ} \mathrm{Cmin}^{-1}$ hasta $\operatorname{los} 200^{\circ} \mathrm{C}$. Se realizó una isoterma durante 15 minutos para luego volver a incrementar la temperatura con una segunda rampa hasta los $300^{\circ} \mathrm{C}$ a $20^{\circ} \mathrm{C} \cdot \mathrm{min}^{-1}{ }^{\circ} \mathrm{C}$, donde se mantuvo durante 5 minutos más. Se utilizó helio como gas transportador con una relación de split 50:1. La detección espectrométrica se realizó con un detector de masas “ 5973 , Agilent Technologies”, (España S.L., Madrid, España). El detector selectivo de masa se programó para detectar masas 
entre 40 y 650 amu. Las muestras de $O$. erraticus fueron pirolizadas a $600^{\circ} \mathrm{C}$ durante $10 \mathrm{~s}$. Los productos de pirólisis fueron identificados obteniendo sus espectros de masas y por comparación de éstos con la librería NIST.98.

\section{RESULTADOS}

En la Tabla 1 se indica el peso, volumen, así como la longitud, anchura y altura para los dos estados estudiados de $O$. erraticus (en ayunas y alimentada). Una observación a bajos aumentos mediante lupa estereoscópica mostró que la rugosidad de la cutícula es mayor en los ejemplares no alimentados mostrando una densa red de protuberancias oscuras, según se observa en la Figura 2, a diferencia de la especie alimentada que muestra una cutícula más lisa, hinchada y clara. Todas las figuras mostradas en el artículo corresponden a una muestra representativa de la población analizada. El área marcada con un círculo muestra la localización donde se hizo el estudio superficial. El eje marcado mediante la línea discontinua muestra la sección última utilizada para la observación en crio-SEM. Un estudio superficial a mayores aumentos mediante microscopía óptica reveló un patrón regular de valles y picos en ambos ejemplares. En cambio las protuberancias observadas son más agudas, estrechas y altas en los ejemplares en ayunas comparados con los alimentados, tal y como se muestra en la Figura 3. Con el fin de demostrar el posible mecanismo de estiramiento durante el engorde, la cutícula de los ejemplares fueron observados mediante crio-SEM tanto superficialmente como en sección transversal. La localización de estos análisis se representan en la Figura 2. Mediante el análisis de las imágenes adquiridas superficialmente se pudo observar que la morfología de las protuberancias es a modo de pliegues cuticulares según se observa en la Figura 4. De forma clara, en el ejemplar alimentado las protuberancias aparecen con menor plegamiento. Además, de la observación en sección transversal de la cutícula se reveló el modo en que los pliegues son empaquetados. La sección del ejemplar en ayunas (Fig. 5a), muestra un cuerpo delgado y plano a diferencia del individuo alimentado (Fig. 5d) que muestra un cuerpo grueso con sus cavidades rebosantes. A mayores aumentos, se comprueba que la altura de los pliegues del individuo en ayunas (Fig. $5 \mathrm{~b}$ y $5 \mathrm{c}$ ) es mayor que su diámetro, resultando $135 \mu \mathrm{m}$ frente a $60 \mu \mathrm{m}$, respectivamente. Por otro lado, en el ejemplar alimentado

TABLA 1. Longitud, anchura, altura y peso de O. erraticus en ayunas y alimentada $(\mathrm{n}=10)$

TABLE 1. Length, width, thickness and weight of $O$. erraticus unfed and fed $(\mathrm{n}=10)$.

\begin{tabular}{llllll}
\hline O. erraticus & $\begin{array}{l}\text { Longitud } \\
(\mathrm{mm})\end{array}$ & $\begin{array}{l}\text { Anchura } \\
(\mathrm{mm})\end{array}$ & $\begin{array}{l}\text { Altura } \\
(\mathrm{mm})\end{array}$ & $\begin{array}{l}\text { Peso } \\
(\mathrm{mg})\end{array}$ & Volumen $\left(\mathrm{mm}^{3}\right)$ \\
\hline Ayunas & $4,08 \pm 0,04$ & $2,3 \pm 0,1$ & $1,08 \pm 0,04$ & $6,0 \pm 1,4$ & $5,16 \pm 0,009$ \\
Alimentada & $5,2 \pm 0,2$ & $3,35 \pm 0,07$ & $1,42 \pm 0,06$ & $13,8 \pm 0,9$ & $12,8 \pm 0,003$ \\
\hline
\end{tabular}

a)

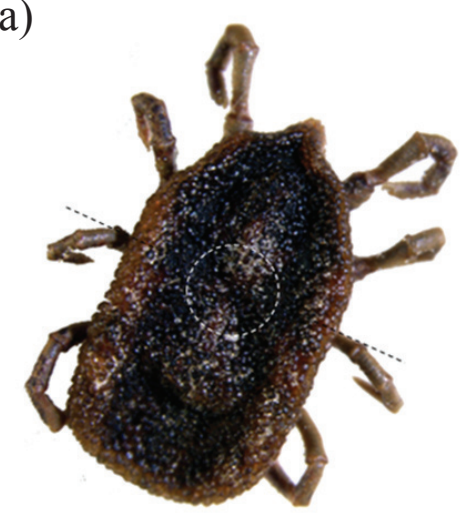

b)

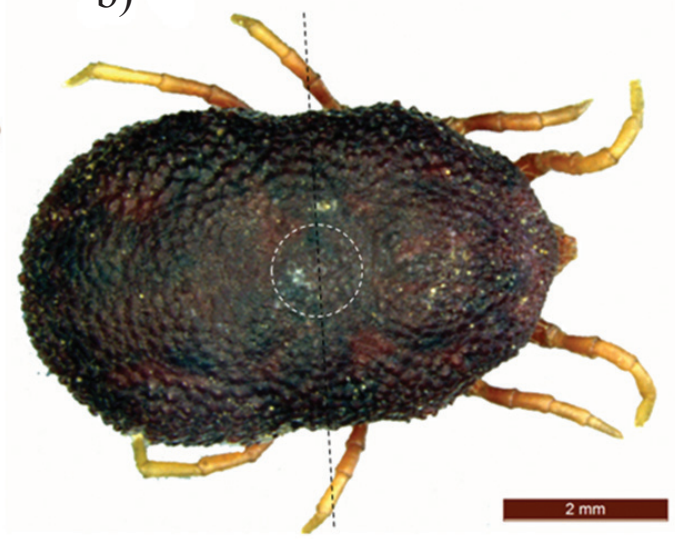

FIgURA 2. Imagen de la parte posterior de un parásito Ornithodoros erraticus adquirida mediante lupa estereoscópica en estado de (a) ayunas y (b) alimentado.

FIGURE 2. Rear image of Ornithodoros erraticus parasite, acquired by stereoscopic magnifying glass in (a) unfed and (b) fed state. 
el pliegue se estira en el plano de la cutícula, resultando una altura de pliegue de $100 \mu \mathrm{m}$ con un diámetro de 150 $\mu \mathrm{m}$ (Fig. 5e). Las imágenes $5 \mathrm{c}$ y $5 \mathrm{f}$ revelan la estructura de células que llenan la cavidad, siendo probablemente las que ejercen la presión necesaria hacia la cutícula, forzando su estiramiento. Un estudio de la sección de la cutícula a mayores aumentos revela la característica estructura de epicutícula superficial cubriendo a la procutícula interna en la que es posible distinguir las microfibrillas de homopolímeros lineales, tal y como muestran las imágenes crio-SEM de la Figura 6. Por otra parte, se utilizó un cromatógrafo de gases acoplado a un espectrómetro de masas para detectar e identificar los productos de pirólisis, respectivamente. En la Figura 7 se muestran los cromatogramas obtenidos de los productos de pirólisis de $O$. erraticus en ayunas y alimentada. Se puede observar la presencia de abundantes productos de pirólisis, encontrándose algunas diferencias en la abundancia relativa, pero en términos generales los cromatogramas revelados por $O$. erraticus en ayunas y alimentada son muy similares. Los principales productos de pirólisis fueron identificados a través de los espectros de masas de cada pico cromatográfico. Como era de esperar, una gran cantidad de los productos de pirólisis encontrados representan productos de pirólisis derivados directamente de proteínas (Steinbrecht \& Stankiewicz 1999). En la Tabla 1 se indican los fragmentos de masas principales de los picos cromatográficos mayoritarios y sus tiempos de retención.
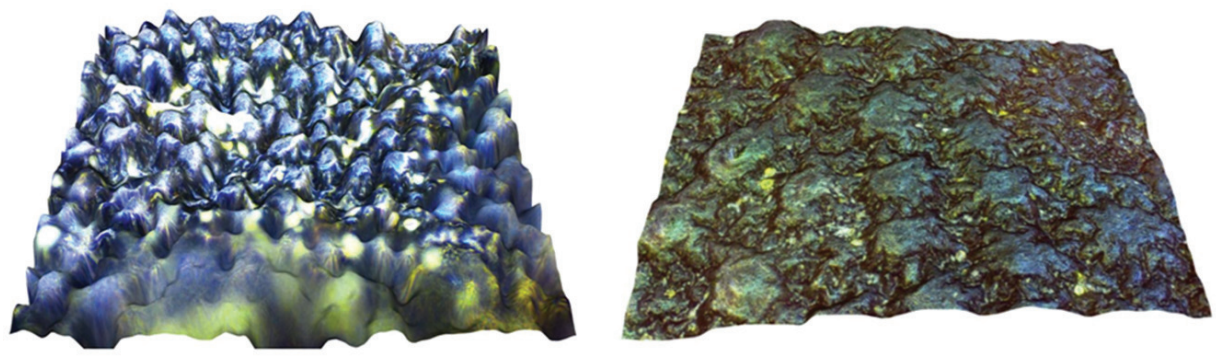

Figura 3. Imágenes de microscopía óptica de la superficie de la cutícula de Ornithodoros erraticus según la zona indicada en Figura 2, de un individuo (a) en ayunas y (b) alimentado.

FIgURE 3. Optical micrographs of Ornithodoros erraticus' cuticle surface according indicated areas in Figure 2, of an individual in (a) unfed and (b) fed state.

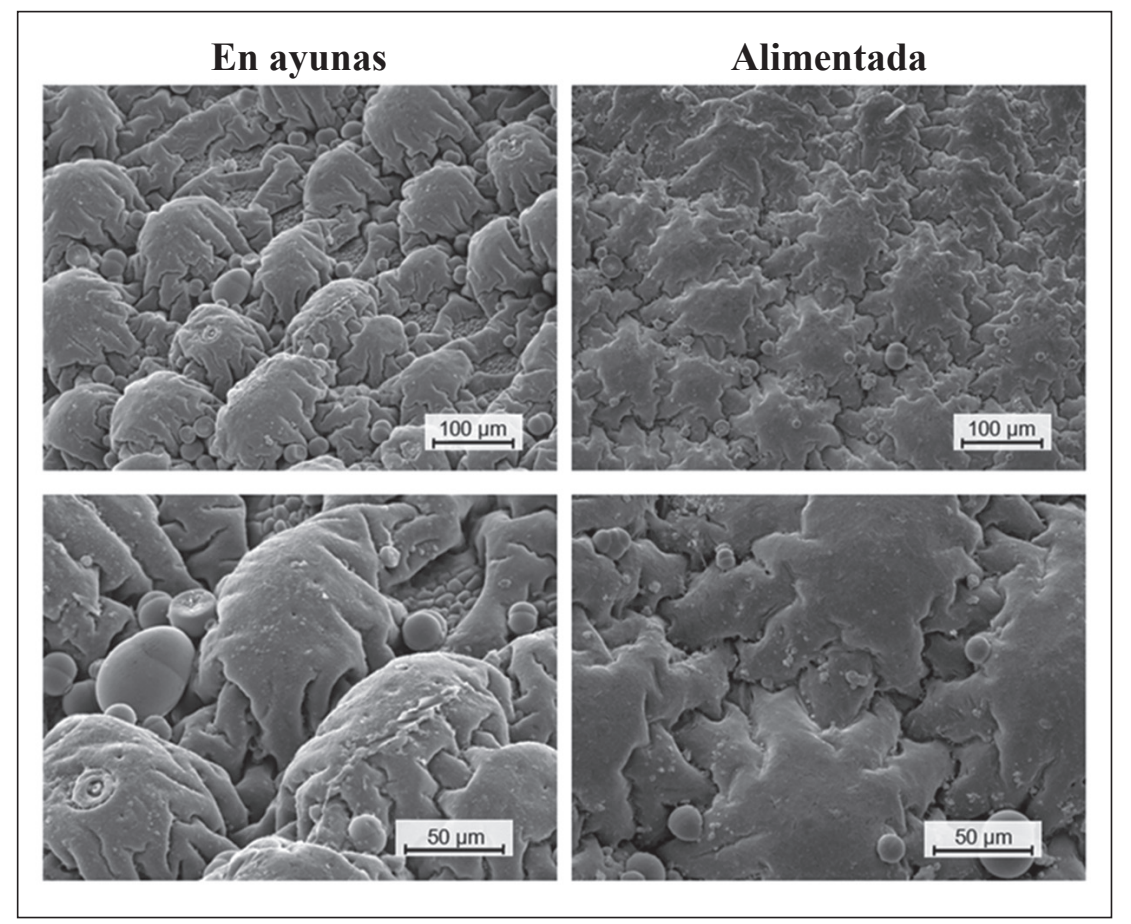

Figura 4. Imágenes crio-SEM de la superficie de la cutícula de Ornithodoros erraticus en estado de ayunas y alimentada.

Figure 4. Crio-SEM images of Ornithodoros erraticus' cuticle surface in unfed and fed state. 


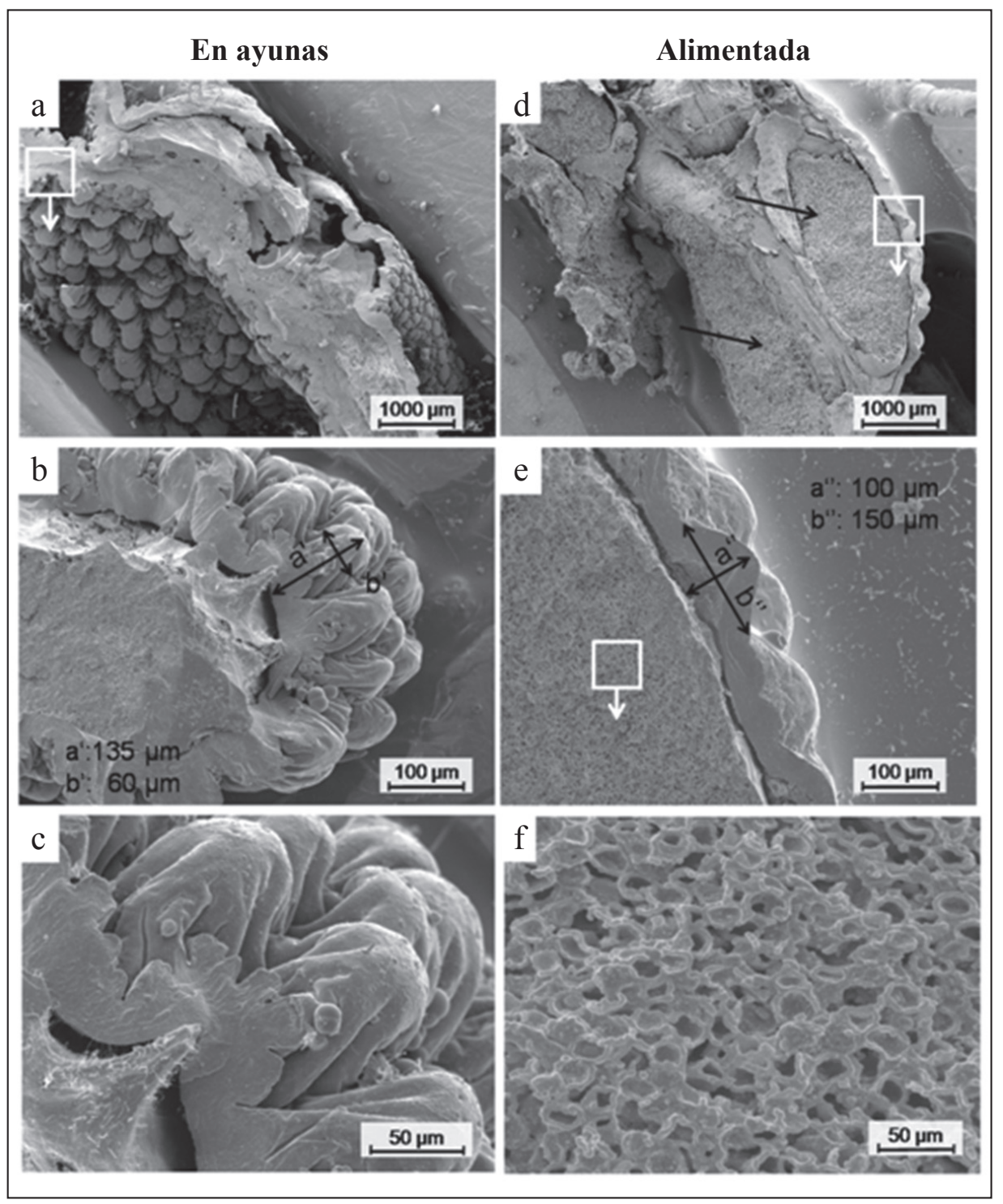

Figura 5. Imagenes crio-SEM a varios aumentos de la sección de Ornithodoros erraticus en ayunas (a,b,c) y alimentada (d,e) con detalle de la estructura de la sangre injerida (f).

Figure 5. Crio-SEM images of Ornithodoros erraticus' section at several magnifications in unfed (a, b, and c) and fed state (d and e).

Los productos mayoritarios encontrados en ambas muestras resultaron los picos que se encuentran a tiempos de retención de 12.40 minutos y 15.56 minutos (Figura 7) y fueron identificados como fenol y metilfenol, respectivamente. La presencia de fenoles refleja la pirólisis del aminoácido tirosina (Flannery et al. 2001). La presencia del aminoácido tirosina ha sido encontrado en cantidades abundantes en la cutícula de Ixodes ricinus (Andersen \& Roepstorff 2005). A tiempo de retención de 18.6 minutos encontramos un producto de pirolisis del ácido láctico (Arrieta et al. 2013), indicando posiblemente la conversión de D-Glucosa en ácido láctico. Los productos a tiempo de retención 32.1 minutos y 32.6 min son productos de degradación de NAG, los cuales revelaron espectros de masas muy similares (Tabla 1) (Drover et al. 2012). El mayor producto de degradación de NAG es el 3-acetoamido-5 acetilfurano (Chen et al. 1998), por lo tanto la presencia de dicho compuesto indica la presencia del monómero de quitina. Así, 3-acetoamido-5 acetilfurano representa el producto mayoritario en el caso de las $O$. erraticus alimentada (pico a tiempo de retención 32.1 minutos), mientras que en el caso de $O$. erraticus en ayunas tiene como producto mayoritario al indol (pico a tiempo de retención 22.06 minutos en la Figura 7-a), indicando la pirólisis del aminoácido triptófano (Flannery et al. 2001). La proporción de indol en $O$. erraticus alimentada es muy inferior a cualquier otro compuesto. 

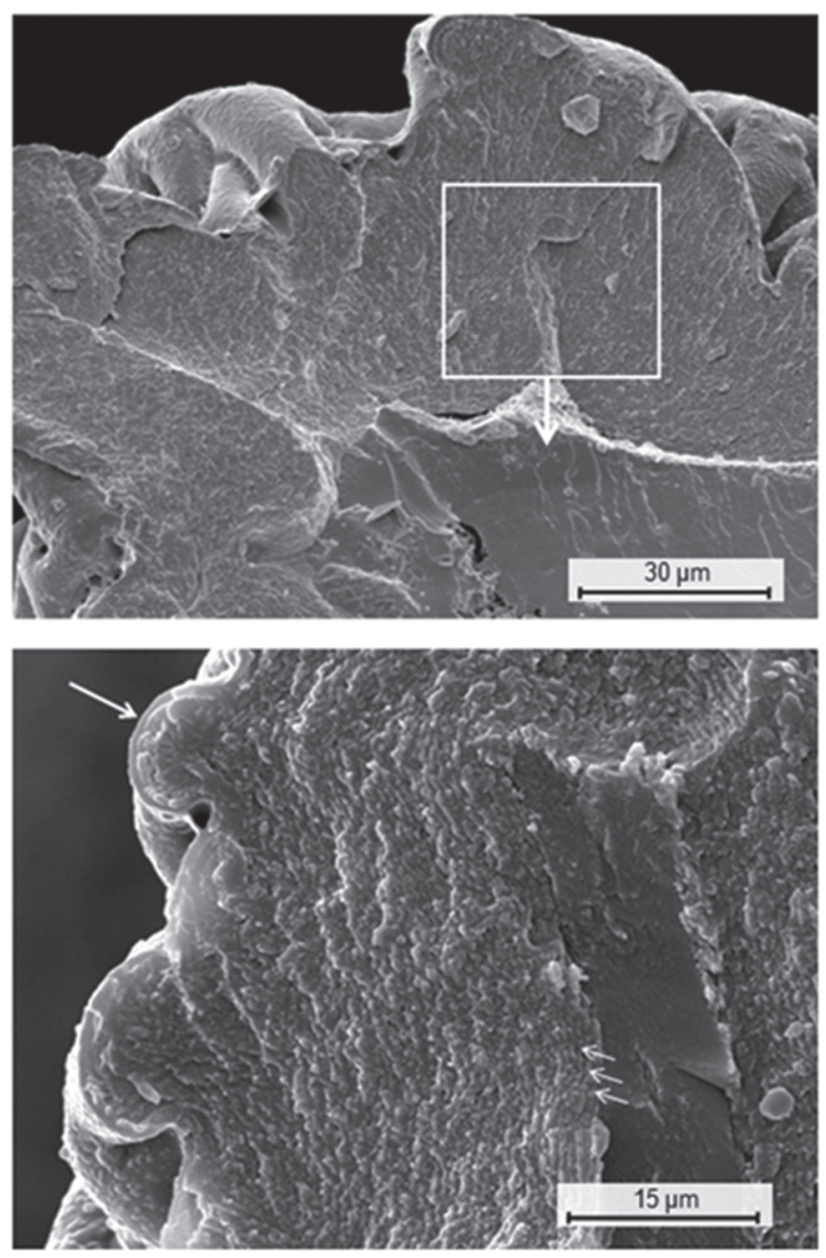

Figura 6. Imagen crio-SEM y detalle de la sección transversal de la cutícula de un ácaro Ornithodoros erraticus. Las flechas indican la epicutícula (superficie exterior) y las microfibrillas que forman la procutícula.

Figure 6. Cryo-SEM image and detail of the cross section of Ornithodoros erraticus' cuticle. The arrows indicate the epicuticle (external surface) and the microfibrils of the procuticle.
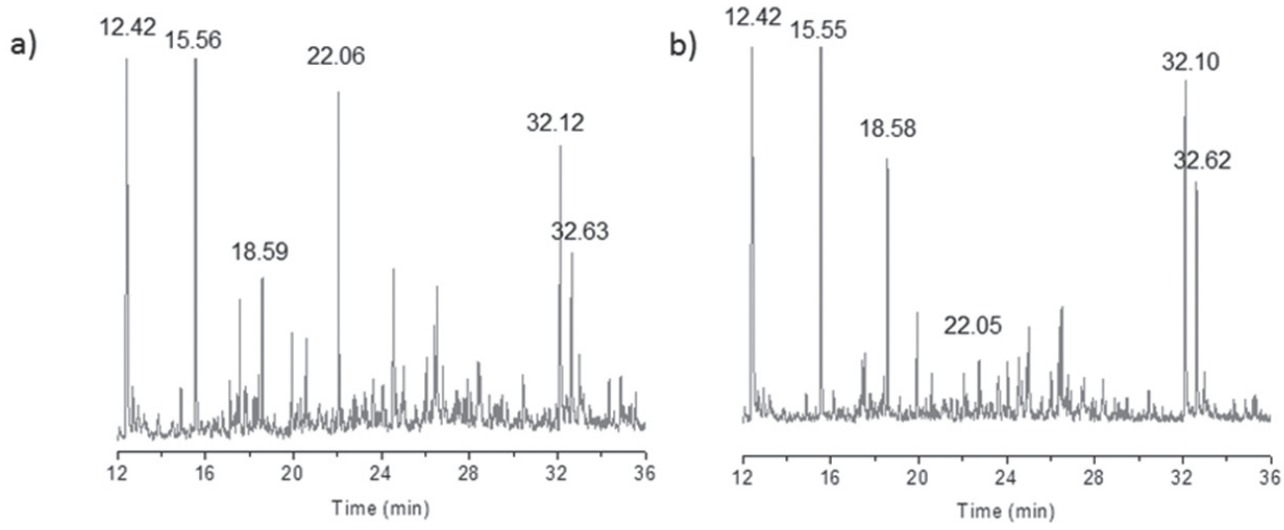

Figura 7. Cromatograma obtenido de la pirólisis a $600^{\circ} \mathrm{C}$ durante $10 \mathrm{~s}$ de $O$. erraticus, a) en ayunas; b) alimentada.

Figure 7. Obtained chromatogram after pyrolysis at $600^{\circ} \mathrm{C}$ for $10 \mathrm{~s}$ of $O$. erraticus in (a) unfed and (b) fed state. 
Tabla 2. Productos mayoritarios obtenidos de la pirólisis de $O$. erraticus a $600^{\circ} \mathrm{C}$ durante $10 \mathrm{~s}$.

TABLE 2. Major pyrolysis products obtained from pyrolysis of $O$. erraticus at $600^{\circ} \mathrm{C}$ for $10 \mathrm{~s}$.

\begin{tabular}{|c|c|c|}
\hline TIEMPO DE RETENCIÓN (min) & COMPUESTO & $\operatorname{MS} \operatorname{Ion}(m / z)^{\mathrm{a}}$ \\
\hline 12.4 & Fenol & $65,66,94$ \\
\hline 15.6 & Metil Fenol & $\mathbf{7 7}, \mathbf{7 9}, \mathbf{1 0 7}, 108$ \\
\hline 18.6 & Lactide & $43,45, \mathbf{5 6}$ \\
\hline 22.1 & Indol & $63,90,117$ \\
\hline 32.1 & 3-Acetoamido 5-acetilfurano & $44,70,97,112,125,168$ \\
\hline 32.6 & 3-Acetoamido 5-acetilfurano & $44,70,97,112,125, \mathbf{1 6 8}$ \\
\hline
\end{tabular}

aprincipal ion (en negrita)

\section{DISCUSIÓN}

Las dimensiones de $O$. erraticus crecen tanto longitudinalmente, como en anchura y altura. Se puede observar que el crecimiento longitudinal no es tan marcado como el encontrado en garrapatas duras (Ixodes ricinus) (Dillinger \& Kesel 2002, Flynn \& Kaufman 2011). Sin embargo, el volumen de Ornithodoros erraticus alimentada es considerablemente mayor que en su estado en ayunas (2,5 veces), mientras que su masa aumentó en 2,3 veces. De la observación morfológica de la cutícula de la Ornithodoros erraticus se puede establecer que el crecimiento de volumen del parásito implica un estiramiento de su cutícula. La morfología en forma de mesetas con una elevada densidad de pliegues permite a la cutícula estirarse en todo el plano cuticular aumentando como consecuencia el volumen del parásito. De este modo los ejemplares alimentados mostraron una superficie más lisa al disminuir la densidad y altura de las protuberancias. Estas observaciones dan a entender un posible mecanismo de estiramiento cuticular. Probablemente las microfibrillas observadas en las imágenes crio-SEM jueguen un papel importante en el estiramiento de la cutícula. Se esperarían tensiones de tracción en el plano cuticular que fuercen a un deslizamiento entre las fibras. Estos posibles deslizamientos podrían ser estudiados en futuros trabajos para esclarecer los mecanismos de deformación plástica a nivel microscópico. Resultados similares fueron reportados por Dillinger y Kesel, quienes estudiaron Ixodes ricinusy concluyendo que la drástica reducción en la dilatación vertical de la cutícula y el aplanamiento de las laminillas es un fenómeno puramente de expansión mecánica (Dillinger \& Kesel 2002). Sin embargo, cabe esperar algún cambio bioquímico y/o de $\mathrm{pH}$ generado por la síntesis de alguna molécula o sustancia que active algún mecanismo por el cual la cutícula pierda rigidez y/o active el estiramiento de la misma. En este sentido, Kaufman y sus colaboradores (2010) reportaron que las propiedades mecánicas de la cutícula de la garrapata podrían ser moduladas por cambios relativamente pequeños en el pH (Kaufman et al. 2010). Encontrar la posible molécula que activa este crecimiento es de especial relevancia puesto que además de clarificar los mecanismos bioquímicos del crecimiento, se podría llegar a bloquearlos mediante vacunas o pesticidas específicos conduciendo a la erradicación o inactividad del parásito. Con este fin, ejemplares de Ornithodoros erraticus alimentados y en ayunas fueron pirolizados. La composición química indica que el aminoácido triptófano se encuentra en mayor proporción en $O$. erraticus en ayunas contribuyendo a la estructura macromolecular rígida del parásito. La menor proporción de este aminoácido encontrada en $O$. erraticus alimentada podría ser causante de la pérdida de rigidez cuticular permitiendo que el aumento de presión isostática generada por la ingesta de alimento provocase el estiramiento de ésta. En este sentido, en un trabajo en curso se están realizando el estudio de la rigidez de la cutícula en estado de ayunas y alimentada mediante la técnica de nanoindentación. Los resultados de estos estudios serán publicados próximamente. Los resultados presentados en el presente trabajo podrían resultar útiles no solo para el estudio de bloqueantes del desarrollo del parasito, sino también como modelo para el desarrollo de nuevos biomateriales. Mediante biomímesis de esta morfología descubierta en forma de pliegues, se podrían generar cutículas sintéticas que fueran capaces de adaptarse a importantes cambios dimensionales. 


\section{CONCLUSIONES}

La microscopía electrónica de barrido mediante criofijación ha sido útil para caracterizar la morfología de la cutícula de ejemplares de Ornithodoros erraticus. Mediante esta técnica se ha determinado que la cutícula se adapta al crecimiento de los parásitos durante su transición a estado alimentado mediante un proceso de estiramiento de un patrón de pliegues en el plano de la cutícula. El análisis químico de la cutícula confirma que durante el estiramiento disminuye la proporción de indol lo que podría provocar una pérdida de rigidez o bien puede ser parte del mecanismo de activación del estiramiento. Los resultados obtenidos muestran un estudio preliminar que contribuyen al esclarecimiento del comportamiento mecanismo y químico de la cutícula de $O$. erraticus en ayunas y alimentada.

\section{AGRADECIMIENTOS}

Los autores desean expresar su agradecimiento a D. Antonio Encinas Grandes por el suministro de Ornithodoros erraticus del área de Salamanca (España) y por su gran aportación a este trabajo mediante la revisión y discusión de los resultados obtenidos. Así mismo, al servicio de microscopía de la Universidad Politécnica de Valencia. MP Arrieta ha sido becada por la Comunitat Valenciana (Grisolía/2011/007). El presente trabajo ha sido financiado por el Ministerio de Educación y Ciencia (MAT2011-28468-C02-02).

\section{REFERENCIAS}

Alonso-Diaz, M. A., Rodriguez-Vivas, R. I., Fragoso-Sanchez, H. \& Rosario-Cruz, R. 2006. Ixodicide resistance of the the Boophilus microplus tick to ixodicides. Archivos De Medicina Veterinaria 38(2): 105-113.

Andersen, S. O. \& Roepstorff, P. 2005. The extensible alloscutal cuticle of the tick, Ixodes ricinus. Insect Biochemistry and Molecular Biology 35(10): 1181-1188.

Arrieta, M., Parres García, F., López Martínez, J., Navarro VidAL, R. \& Ferrándiz, S. 2012. Pyrolysis of bioplastic waste: Obtained products from Poly (Lactic acid), PLA. DYNA 87(4):395-399.

Arrieta, M. P., Parres, F., López, J. \& Jiménez, A. 2013. Development of a novel pyrolysis-gas chromatography/ mass spectrometry method for the analysis of poly(lactic acid) thermal degradation products. Journal of Analytical and Applied Pyrolysis 101: 150-155.

BARANDIKa IzA, J. F. 2010. Las garrapatas exófilas como vectores de agentes zoonóticos: estudio sobre la abundancia y actividad de las garrapatas en la vegetación, e investigación de la presencia de agentes patógenos en garrapatas y micromamíferos. Departamento de Sanidad Animal. León, Universidad de León. Dr: 273.

Berrios, P. 2002. Artropodos Asociados a suelo de renovales de
Nothofagus Obliqua (Mirb.) Oersted (Fagacea) En la zona costera de la VIII Region. Gayana (Concepción) 66: 1-6.

Chen, J. H., Wang, M. F. \& Ho, C. T. 1998. Volatile compounds generated from thermal degradation of $\mathrm{N}$-acetylglucosamine. Journal of Agricultural and Food Chemistry 46(8): 3207-3209.

Dillinger, S. C. G. \& Kesel, A. B. 2002. Changes in the structure of the cuticle of Ixodes ricinus L. 1758 (Acari, Ixodidae) during feeding. Arthropod Structure and Development 31(2): 95-101.

Drover, M. W., Omari, K. W., Murphy, J. N. \& Kerton, F. M. 2012. Formation of a renewable amide, 3-acetamido5 -acetylfuran, via direct conversion of N-acetyl-Dglucosamine. Rsc Advances 2(11): 4642-4644.

Encinas Grandes, A. 1999a. Artrópodos. Parasitología Veterinaria. Cordero del Campillo, M. and Rojo Vázquez, F. A. Madrid, McGraw Hill Interamericana.

Encinas Grandes, A., Oleaga Pérez, A. \& Pérez Sánchez, R. 1999b. Garrapatas Duras. Parasitologia Veterinaria. Cordero del Campillo, M. and rojo Vázquez, F. A. Madrid, McGraw Hill Interamericana: 420-429.

Encinas Grandes, A., Pérez Sánchez, R. \& Oleaga Pérez, A. 1999c. Ornitodorosis e ixodidosis. Parasitologia Veterinaria. Cordero del Campillo, M. and Rojo Vázquez, F. A. Madrid, McGraw Hill Interamericana.

Flannery, M. B., Stott, A. W., Briggs, D. E. G. \& Evershed, R. P. 2001. Chitin in the fossil record: identification and quantification of D-glucosamine. Organic Geochemistry 32(5): 745-754.

FlynN, P. C. \& Kaufman, W. R. 2011. Female ixodid ticks grow endocuticle during the rapid phase of engorgement. Experimental and Applied Acarology 53(2): 167-178.

Hackman, R. H. 1982. Structure and function in tick cuticle. Annual Review of Entomology 27: 75-95.

Hackman, R. H. \& Goldberg, M. 1987. Comparative study of some expanding arthropod cuticles: The relation between composition, structure and function. Journal of Insect Physiology 33(1): 39-50.

Iannacone, J. \& Ayala, L. 2004. Census of Ornithodoros amblus chamberlin (Acarina: Argasidae) in mazorca island, Lima, Peru. Parasitología Latinoamericana 59: 56-60.

Kaufman, W. R., Flynn, P. C. \& Reynolds, S. E. 2010. Cuticular plasticization in the tick, Amblyomma hebraeum (Acari: Ixodidae): possible roles of monoamines and cuticular pH. Journal of Experimental Biology 213(16): 28202831.

Lomakin, J., Huber, P. A., Eichler, C., Arakane, Y., Kramer, K. J., Beernan, R. W., Kanost, M. R. \& Gehrke, S. H. 2011. Mechanical Properties of the Beetle Elytron, a Biological Composite Material. Biomacromolecules 12(2): 321-335.

Muñoz, L., Aguilera, M. \& Casanueva, M. E. 2003. Prevalencia e intensidad de ectoparásitos asociados a Tadarida brasiliensis (Geoffroy Saint-Hilaire, 1824) (Chiroptera: Molossidae) en Concepcion. Gayana (Concepción) 67: 1-8.

Muñoz, L. E. \& Casanueva, M. E. 2001. Present state of the knowledge of ticks (Acari. Ixodida) associated to Canis familiais L. Gayana 65(2): 193-210. 
OJeda Sahagún , J. L. 1997. Métodos de microscopía electrónica de barrido en biología. Santander, Universidad de Cantabria.

Parola, P. \& Didier, R. 2001. Ticks and tickborne bacterial diseases in humans: An emerging infectious threat. Clinical Infectious Diseases 32(6): 897-928.

Stankiewicz, B. A., Poinar, H. N., Briggs, D. E. G., Evershed,
R. P. \& PoInar, G. O. 1998. Chemical preservation of plants and insects in natural resins. Proceedings of the Royal Society B-Biological Sciences 265(1397): 641647.

Steinbrecht, R. A. \& Stankiewicz, B. A. 1999. Molecular composition of the wall of insect olfactory sensilla - the chitin question. Journal of Insect Physiology 45(8): 785 790 .

Recibido: 06.11.12

Aceptado: 11.03.13 